\title{
The Association Between the Postnatal Environment and Behavioral Sexual Dimorphism in Pre-school Children in Single-Child Families in Qingdao, China
}

\author{
Bingling Wang ${ }^{1}$, Qian Zhang ${ }^{2}$, Guochang Feng ${ }^{3}$, Zhongqing Sun ${ }^{4}$, Lijuan Wang ${ }^{5}$, Xiao Jia ${ }^{2}$, \\ Shutao Pang ${ }^{6}$, Ruqin Gao ${ }^{1, *}$ \\ ${ }^{1}$ Qingdao Centers for Disease Control and Prevention, Qingdao, China \\ ${ }^{2}$ Department of Child Health Care, Maternity and Child Care Center of Huangdao District of Qingdao, Qingdao, China \\ ${ }^{3}$ Business Management Office of Qingdao Centers for Disease Control and Prevention, Qingdao, China \\ ${ }^{4}$ Department of Nutrition and Food Hygiene of Qingdao Centers for Disease Control and Prevention, Qingdao, China \\ ${ }^{5}$ Microorganism and Parasite Detection Laboratory of Qingdao Centers for Disease Control and Prevention, Qingdao, China \\ ${ }^{6}$ Qingdao Blood Center, Qingdao, China
}

Email address:

gaoruqin@yeah.net (Ruqin Gao)

${ }^{*}$ Corresponding author

To cite this article:

Bingling Wang, Qian Zhang, Guochang Feng, Zhongqing Sun, Lijuan Wang, Xiao Jia, Shutao Pang, Ruqin Gao. The Association Between the Postnatal Environment and Behavioral Sexual Dimorphism in Pre-school Children in Single-Child Families in Qingdao, China. Science Journal of Public Health. Vol. 4, No. 6, 2016, pp. 500-505. doi: 10.11648/j.sjph.20160406.24

Received: November 8, 2016; Accepted: December 2, 2016; Published: December 5, 2016

\begin{abstract}
Previous studies have shown that prenatal exposure to chemical or non-chemical endocrine disruptors can alter children's sexually dimorphic behavior. To date, no study has examined the possible influence of postnatal-environmental factors on the gender-specific play behavior of childhood. We hypothesized that the postnatal environment in early childhood might also influence the sexual dimorphism of the brain. Parents or guardians of pre-school children completed questionnaires about their postnatal living environment. The Pre-School Activities Inventory (PSAI) was used to measure sexually dimorphic play behavior. Sex-stratified analyses revealed that the generosity $(\beta=0.085, \mathrm{p}<0.05)$ and education $(\beta=0.142, \mathrm{p}<0.01)$ of fathers predicted higher feminine scores in girls and lower feminine $(\beta=-0.144, \mathrm{p}<0.001)$ or higher masculine scores $(\beta=0.129, \mathrm{p}<0.01)$ in boys. Indices of chemical exposure (i.e., indoor decoration, biomass pollution, hand cleanliness, and passive smoking) had an association with PSAI scores in both girls and boys. Patterns of sensory processing, including tactile and proprioceptive senses scores and sensory underresponsivity scores, also were associated with PSAI scores. An association of PSAI scores with taking drugs during pregnancy and gestational nutrition was evident only in boys. Our results indicate that a social environment of parental guidance and an indoor environment of exposure to chemical are associated with behavioral sexual dimorphism. Children's patterns of sensory processing also contributed to behavioral sexual dimorphism. Information about the gestational environment also should be considered when studying the development of sexual dimorphism in boys.
\end{abstract}

Keywords: Postnatal Exposure, Play Behavior, Pre-school Activities Inventory, Sexual Dimorphism, Neurodevelopment, Preschool

\section{Introduction}

Previous studies have shown that prenatal exposure to endocrine-disrupting chemicals can alter children's neurodevelopment. Recently, the brain's sexual dimorphism also has been recognized to be the target of the neurodevelopmental toxicants. Prenatal exposure to phthalates [1], polychlorinated biphenyls (PCBs), dioxins [2, 3], and soy [4] have been reported to cause increased as well as decreased masculine behavior in boys and decreased feminine behavior in girls. Furthermore, a recent study revealed that non-chemical exposures, such as prenatal 
life-event stress can cause the same effects as those caused by the endocrine-disrupting chemicals [5].

Zambrano and colleagues reviewed previous studies and concluded that the brain's sexual differentiation was complete in the latter weeks of the gestational period in males, whereas it was not completed in females until 1-3 years of age [6]. Given that there also is a critical period of vulnerability for the developing nervous system between birth and at least two years of age, we hypothesize that the postnatal environment in early childhood also might influence the brain's sexual dimorphism. Recent reports have provided some evidence for our hypothesis. For example, Winneke et al. [2] investigated the association of prenatal and postnatal exposure to polychlorinated dibenzo-p-dioxins and dibenzofurans (PCDD/Fs) and PCBs in maternal blood or early breast milk with the parent-reported sexually dimorphic behaviors of children aged 6-8 years. The study found that the levels of $\mathrm{PCDD} / \mathrm{Fs}$ and PCBs in breast milk, which was collected within the first 3 weeks following birth, were more strongly associated with sexual dimorphic behavior than the levels measured in maternal blood that was collected between weeks 28 and 43 of pregnancy. In addition, the study found a significant association between femininity scores on the Pre-School Activities Inventory (PSAI) and total postnatal milk intake in their statistical model. A study by Vreugdenhil et al [3]. found that higher exposure to PCBs in breast milk was associated with more masculine behavior in girls.

The integration of sensory information from the body and the environment is essential for almost every human activity and involves the brain selecting, inhibiting, comparing, and associating sensory information [7] and permits the planning and production of organized behavior [8]. The prevalence of sensory processing disorder (SPD) among children with developmental delays is estimated to be as high as $40-88 \%$ [9]. Sensory processing patterns can be identified effectively using a sensory profile and can be indicative of childhood disabilities and developmental delays.

In this study, we collected information about the child's living environment from the child's parents or guardians to examine the association of the postnatal environment with behavioral sexual dimorphism and sensory processing patterns in kindergarten children.

\section{Methods}

\subsection{Recruitment of Participants}

Four kindergartens were randomly sampled from 11 public kindergartens of a district of Qingdao, China. A total of 2,080 questionnaires were distributed in these four kindergartens, and 1,731 were returned. Of these, 200 questionnaires were excluded because they had insufficient information on the PSAI or SPD scores. Totally 192 children were not from a single-child family. Given that presence and sex of siblings in the home might be the import confounder of the play behavior [10], these 192 children were excluded. This left 1,339 participants for inclusion in the analyses. This study was approved by the Ethics Committee and Institutional Review Board of Qingdao Centers for Disease Control and Prevention. All participants provided their written informed consent before inclusion in the study.

\subsection{Questionnaire}

The practicability of the self-administered questionnaire was tested with the parents of 100 children in one public kindergarten of the sampled district. The questions were rearranged or changed as needed, based on the pilot test, to ensure that the questionnaire was intelligible. The final questionnaire was taken back home by the children, and the guardians who had lived with the children for at least the past 3 months and knew their health condition and day-to-day behaviors were requested to complete the questionnaire, with reference to the parents if necessary.

The questionnaire included 8 parts: 1) general information about their child, 2) gestational information, 3) the family care environment, 4) the indoor environment of the home, 5) the outside environment of the home, 6) the indoor environment of the kindergarten, 7) the disease history of the child, and 8) the severity of sensory processing dysfunction (SPD). Most of these questions were based on the "Questionnaire for national investigation for Sick Building Syndrome and its potential risk factors in Japan" [11, 12]. The questions about gestation, asked about drugs taken, passive smoking, and nutrition during pregnancy, as well as the mode of delivery. The family-care environment included information about family income, the educational levels of the guardian, mother, and father, the care mode, or style, of the parents, the child's communication time with parents or guardian, and the closeness of the relationship between the child and parents or guardian. Information about the indoor environment of the home included the decorative materials, the nature of the furniture, the ventilation, smoking behavior within the dwelling, frequency of cleaning by vacuuming or other methods, and indicators of dampness (e.g., the presence of dew condensation, mold growth, or water stains) [13]. Given that some semi-volatile organic compounds are released from domestic electric appliances, the number of electric appliances was recorded. Questions about the outside environment mainly concerned exposure to pollution, including whether the house was near a major street or other known pollution sources. Information about plastic and other chemical odors, uncomfortable feeling or symptoms, and educational mode in the kindergarten were collected. Disease history included any history of allergies, multiple chemical sensitivity (MCS), and newborn diseases, including kernicterus, meningitis, malnutrition, asphyxia, and hypoglycemia, which might influence the development of the nervous system. Other disease-related information included major injuries, such as fractures, infantile convulsions, brain traumas, wheezing, coughing for a long period, frequently catching colds, and frequently taking antibiotics. For the MCS, each child gained one point on the MCS score by setting scale scores to all subjective complaints [14]. Pollution sources near the house were identified as gas station, factory, household waste 
incineration plant, and other facilities that could cause environmentally related complaints.

The SPD profile is a 60 -item guardian questionnaire designed to provide information about the child's ability to process sensory information. The SPD profile was first translated and standardized by the Institute of Mental Health, Beijing Medical University [15]. After several revised versions, now it consists of 6 subtypes of senses: vestibular (10 items), underresponsivity (10 items), tactile (14 items), dyspraxia (11 items), visual (5 items) and proprioceptive (10 items) [16]. The test-retest reliability is reported to be $0.47-0.73$, the split-half reliability is $0.68-0.77$, and the homogeneity reliability is $0.49-0.9$ [15]. Responses to the items of the SPD profile correspond to relative frequency of the occurrence of the child's behavior, according to the guardian. The answer to each item is reported on a 5-point, Likert-type scale, ranging from never (scored as 1) to always (scored as 5).

\subsection{Pre-school Activities Inventory (PSAI)}

Parents or guardians were asked to complete the PSAI [17], a validated, standardized tool that is designed to assess sexually dimorphic behavior. The PSAI consists of 24 items (12 considered "feminine" and 12 "masculine"), which are grouped into three aspects of play behavior: preferred toys, activities, and behavioral characteristics. Answers are reported on a 5-point, Likert-type scale ranging from "never" to "very often." The profile consists of three subtypes: a feminine score (the sum of girl's typical frequency-weighted items), a masculine score (the sum of boy's typical frequency-weighted items), and a composite score (feminine score minus masculine score) [3].

\subsection{Statistical Analysis}

The raw subtype scores of the SPD profile were transformed into $\mathrm{T}$ standard scores. Therefore, higher scores indicate more typical behavior, whereas lower scores indicate a difference in behavior compared to children without dysfunction.

Descriptive and summary statistics for relevant variables were examined first, and then bivariate associations were tested. We assumed that there would be sex differences in PSAI scores; therefore, all analyses (bivariate and multivariate) were stratified by sex. Spearman's rho correlations were performed on the continuous variables to test their possible associations with PSAI scores. Student's $t$ test or one-way analysis of variance (ANOVA) was used to test the association of the PSAI scores with the categorical variables. A linear trend was tested for the variables analyzed by one-way ANOVA. If the test resulted in a $p$ value $<0.05$, the variable was selected for inclusion in multivariate linear regression models.

The Kolmogorov-Smirnov test showed that the PSAI scores of boys and girls were distributed normally. Three multivariate linear regression models were performed for each sex to examine the association between possible risk factors and the: 1) feminine PSAI scores, 2) masculine PSAI scores, and 3) composite PSAI scores. All independent variables were entered into the models backwards; the $p$-value for inclusion was 0.05 and the $p$-value for removal was 0.10 . A $p<0.05$ was considered to be statistically significant. All analyses were performed using IBM SPSS Statistics version 21 (IBM Corp, Somers, NY). The results of the regression analyses for the association between the possible risk factors and the PSAI scores are presented as adjusted regression coefficients $(\beta)$, together with their $95 \%$ confidence intervals (CIs) and Wald $p$-values.

\section{Results and Discussion}

In total, data from 989 (48.0\%) girls and 1070 (52.0\%) boys were included into the final analyses. We assume that boys and girls do present with some characteristics of the opposite sex. In Table 1, the mean differences between boys and girls for the respective score values were highly significant $(p<0.001)$, and the boys $(37.4 \pm 8.1)$ had higher masculine scores than the girls $(27.4 \pm 5.8)(p<0.001)$. Hence, as expected, the composite scores were higher for the girls (14.2 \pm 8.0$)$ than for the boys $(-10.0 \pm 8.1)(p<0.001)$. Our results are close to the PSAI scores of our another pilot study $(p>0.05)$ [18], but lower than another Chinese study in $1995(p<0.05)$ [19]. Table 1 showed that our average St of the boys was lower, but Sf was higher, than those reported in other countries, $p<0.05$. Our Sm was higher than those of the Germany [2] and Netherland [3], but lower than the USA [1], $p<0.05$. For the girls, no difference was found between our St, Sf and Sm value and those of the USA $(p>0.05)$, but our St was lower and Sf and Sm were higher than those of the other countries $(p<0.01)$. Whether these differences are the truth or caused by the PSAI needs further investigation.

Table 1. Comparison of the PSAI score in the present study and those in the literature (Mean $\pm S D)$.

\begin{tabular}{|c|c|c|c|c|c|c|c|c|c|c|c|}
\hline \multirow{2}{*}{ Countries } & \multirow{2}{*}{ year } & \multirow{2}{*}{ Age (y) } & \multirow{2}{*}{ N (boy/girl) } & \multicolumn{4}{|l|}{ boy } & \multicolumn{4}{|l|}{ girl } \\
\hline & & & & St & Sf & Sm & Sc & St & Sf & Sm & Sc \\
\hline Present study & 2015 & $3-6$ & $1070 / 989$ & $59.8 \pm 9.0$ & $27.4 \pm 5.8$ & $37.4 \pm 8.1$ & $-10.0 \pm 8.1$ & $32.8 \pm 8.9$ & $41.9 \pm 6.4$ & $27.7 \pm 7.0$ & $14.2 \pm 8.0$ \\
\hline Germany $^{[2]}$ & 2014 & $6.6 \pm 0.5$ & $61 / 60$ & $67.1 \pm 8.0$ & $11.2 \pm 4.5$ & $28.3 \pm 6.0$ & $-17.1 \pm 7.3$ & $36.5 \pm 8.4$ & $28.2 \pm 6.9$ & $17.6 \pm 4.8$ & $10.7 \pm 7.6$ \\
\hline $\mathrm{USA}^{[1]}$ & 2010 & $4-5$ & $74 / 71$ & $65.9 \pm 8.6$ & $24.7 \pm 6.1$ & $40.8 \pm 5.9$ & & $31.4 \pm 8.7$ & $43.2 \pm 5.5$ & $27.9 \pm 5.7$ & \\
\hline $\mathrm{UK}^{[28]}$ & 2008 & $2.5-8.0$ & $2726 / 2775$ & $64.2 \pm 8.8$ & & & & $35.1 \pm 9.4$ & & & \\
\hline China $^{[11]}$ & 1993 & $3-6$ & $203 / 175$ & $66.0 \pm 9.6$ & & & & $37.3 \pm 10.9$ & & & \\
\hline $\mathrm{UK}^{[9]}$ & 1993 & $2-6$ & $1166 / 926$ & $61.7 \pm 9.4$ & & & & $38.7 \pm 9.7$ & & & \\
\hline
\end{tabular}


Table 2. Association between influence factors and PSAI scores in multivariable analyses.

\begin{tabular}{|c|c|c|c|c|c|c|}
\hline \multirow{2}{*}{ Factors } & \multicolumn{2}{|l|}{ Sf } & \multicolumn{2}{|l|}{ Sm } & \multicolumn{2}{|l|}{ Sc } \\
\hline & $\beta(95 \% \mathrm{CI})$ & $P$ & $\beta(95 \% \mathrm{CI})$ & $P$ & $\beta(95 \%$ CI $)$ & $P$ \\
\hline \multicolumn{7}{|l|}{ Girls } \\
\hline Age & & & & & $0.080(0.053,1.273)$ & 0.023 \\
\hline Father's education & $0.152(0.570,2.090)$ & 0.001 & $0.095(0.058,1.565)$ & 0.036 & & \\
\hline Father's care style & $0.075(0.033,0.885)$ & 0.033 & & & $0.090(0.144,1.146)$ & 0.025 \\
\hline Communication time P. & & & $0.073(0.040,2.782)$ & 0.045 & & \\
\hline No. of furniture with B.M. & $0.063(-0.023,0.745)$ & 0.076 & & & $0.090(0.071,0.960)$ & 0.034 \\
\hline Moisture score & & & $0.124(0.323,1.477)$ & 0.004 & $-0.078(-1.230,-0.071)$ & 0.026 \\
\hline Tactile senses score & & & $-0.111(-0.120,-0.011)$ & 0.017 & $0.094(0.013,0.130)$ & 0.040 \\
\hline $\begin{array}{l}\text { Proprioceptive senses S. } \\
\text { Boys }\end{array}$ & $-0.088(-0.125,-0.021)$ & 0.014 & $0.085(-0.002,0.120)$ & 0.060 & $-0.165(-0.200,-0.067)$ & $<0.001$ \\
\hline Age & $-0.098(-0.964,-0.089)$ & 0.020 & & & $-0.123(-1.564,-0.260)$ & 0.004 \\
\hline Father's age & $0.100(0.020,0.251)$ & 0.026 & & & & \\
\hline Father's education & & & $0.130(0.530,2.549)$ & 0.003 & $-0.142(-0.710,-2.611)$ & 0.001 \\
\hline Father's care style & $0.174(0.169,0.870)$ & 0.006 & $0.070(-0.034,1.030)$ & 0.065 & & \\
\hline Drugs taken in gestation & & & $0.089(0.329,5.500)$ & 0.025 & $-0.071(-4.500,0.420)$ & 0.089 \\
\hline Gestational nutrition & & & $-0.100(-3.728,0.151)$ & 0.079 & & \\
\hline Deliver mode & $-0.125(-0.810,-0.169)$ & 0.003 & & & $-0.075(-0.890,0.051)$ & 0.080 \\
\hline Chemical flavor in kind. & $-0.391(-5.414,-0.265)$ & 0.031 & & & $-1.118(-7.594,-1.079)$ & 0.013 \\
\hline Education mode in kind. & $0.093(0.138,2.282)$ & 0.027 & $0.101(0.341,3.518)$ & 0.017 & & \\
\hline Score of MCS & $0.122(0.056,0.287)$ & 0.004 & & & & \\
\hline Hand cleanliness & $-0.129(-2.428,-0.502)$ & 0.003 & & & $-2.152(-3.897,-1.157)$ & $<0.001$ \\
\hline Passive smoking & $0.081(-0.046,2.168)$ & 0.06 & $1.232(0.910,4.211)$ & 0.003 & & \\
\hline Family income & & & $0.099(0.028,1.330)$ & 0.036 & & \\
\hline Underresponsive senses $\mathrm{S}$. & $-0.019(-0.081,-0.012)$ & 0.042 & $-0.107(-0.177,-0.002)$ & 0.039 & & \\
\hline Proprioceptive senses S. & & & $0.160(0.051,0.212)$ & 0.001 & $-0.144(-0.174,-0.051)$ & 0.001 \\
\hline
\end{tabular}

Early postnatal communication with parents and guardians may influence sexually dimorphic behaviors. Father's generosity $(\beta=0.075, p<0.05)$ and level of education $(\beta=$ $0.152, p<0.05)$ predicted higher feminine scores in the girls, whereas father's education $(\beta=0.095, p<0.05)$ and greater communication time with parents $(\beta=0.073, p<0.05)$ predicted higher masculine scores in the girls. Father's education and care style had similar "net effects" on the PSAI scores of the boys as it did on the scores of the girls, with education $(\beta=0.130, p=0.003)$ and generosity $(\beta=0.070, p=$ 0.065 ) being associated with more masculine behavior in the boys, and generosity $(\beta=0.174, p=0.006)$ being associated with more feminine behavior (Table 2). Ordinary care of children by tolerant parents or guardians seems to benefit the development of brain sexual dimorphism. Parents who are older or better educated might know more about how to communicate with a child, so that a closer relationship can be established between parent and child. A generous parent also appears to make a beneficial contribution to sexual development. Recent human studies have established that early child-maternal relationships are associated with differences in brain structure measured at school age [20,21]. It is interesting that only the father's style of care and level of educational were associated with sexually dimorphic behaviors in the multivariate analyses, although the characteristics of both parents had significant associations with PSAI scores in the univariate analyses. Whether this is because the fathers having more influence on the child than the mothers with the same educational level or generous style of care needs further investigation.

Exposure to chemical factors in the early postnatal environment is likely to influence gender-typical behavior.
Indoor decorations can cause aldehyde and VOC pollution, and some aldehydes and VOCs, like formaldehyde and toluene, have been found to be neurodevelopmental toxicants [22]. It is well known that polycyclic aromatic hydrocarbons (PAHs) are the main pollutants found in the kitchen, and prenatal and postnatal PAH exposure have been associated with adverse neurodevelopmental effects [23-25]. Chemical odor was used as the indicator of plastic furnishings in the kindergarten, since plastic furnishings contain a relatively large amount of additives, such as plasticizer and flame retardants. These additives are not covalently bound to the polymers with which they are mixed and they can migrate from the materials to the outside [26]. Most of these additives, including phthalates and polybrominated diphenyl ethers, are endocrine disrupting chemicals and have been found to cause cognitive damage or neurobehavioral changes [27-30]. We used hand cleanliness as an indirect index of exposure to metal or organic chemicals in children because the hand-to-mouth route has been found to be a major pathway for the ingestion of chemicals by children, especially in pre-school children and toddlers [31]. Our multivariate analysis results showed that the number of furniture with toxic materials releasing more volatile or semi-volatile organic compounds was positively associated with the composite score of the girls $(\beta=0.090, p=$ 0.034). Chemical odors in kindergarten, having dirty hands, and passive smoking during pregnancy were associated with more masculine behavior in the boys, while boys who reported sensitive to multiple chemicals showed more feminine behavior (Table 2). Higher moisture scores predicted higher masculine behavior in girls $(\beta=0.124, p=0.004)$ and lower composite scores $(\beta=-0.0788, p=0.026)$. In line with our results, Winneke et al. [2] reported that early postnatal 
exposure to PCBs and dioxins in milk was associated with less masculinized play in girls, and more masculinized play in boys, as assessed by the composite score. However, an earlier study by Vreugdenhil et al.[3] reported that early postnatal exposure to PCBs in milk was related to more masculinized play in girls. Prenatal exposure to phthalate, another antiandrogen chemical, has been reported to reduce masculinized play in boys [1], and early postnatal eating of soy has been associated with reduced feminized play in girls [4]. There are obvious discrepancies in the effects of different chemicals among studies. The different associations observed for feminine behavior [(anti) estrogenicity] and masculine behavior [(anti) androgenicity] could be interpreted as reflecting the differential disruption of endocrine activities by various chemicals.

We examined the association between SPD and sexually dimorphic behaviors. Other childhood disorders and developmental delays, such as Autism Spectrum Disorder (ASD) and Attention Deficit Hyperactivity Disorder (ADHD), have been shown to entail be characterized by atopic sensory processing patterns $[9,32,33]$. Children with ASD have sensory dysfunctions in audition, vision, taste/smell, and tactile sensory discrimination [32]. The sensory dysfunctions in ADHD are visual, tactile, and vestibular sensory discrimination [32]. We found simultaneous tactile and proprioceptive sensory discrimination disorders and sensory modulation disorders (i.e., sensory underresponsivity) that were associated with sexually dimorphic behavior. Lower proprioceptive-sense scores predicted lower feminine behavior scores $(\beta=-0.088, p=0.014)$ and lower composite scores $(\beta=-0.165, p<0.001)$ in girls, whereas lower tactile-senses scores predicted higher masculine behavior scores $(\beta=-0.111, p=0.017)$ and higher composite scores $(\beta$ $=0.094, p=0.040)$. Lower scores for underresponsive senses $(\beta=-0.019, p=0.042)$ and higher scores for proprioceptive senses $(\beta=0.160, p=0.001)$ were associated with higher masculine scores in the boys. Sensory underresponsivity usually leads to poor tactile and proprioceptive discrimination, i.e. children with sensory underresponsivity often have concomitant sensory discrimination disorder [34]. This implies that children with ASD, ADHD, and sex-related behavioral disorders are similar in terms of sensory processing issues. Simultaneously, the SPD-related developmental delay seems associated with sex-related behavioral disorder. Our results confirmed this indirectly. Older age was associated with more feminine behavior in the girls (composite score: $\beta=$ $0.080, p=0.023$ ) and more masculine behavior in the boys (composite score: $\beta=-0.123, p=0.004$ ) (Table 2). Thus, it is difficult to conclude that developmental delays or SPD contributed to sex-related behavioral disorders.

Another important finding of our study is that gestational risk factors, such as gestational nutrition, taking drugs, and passive smoking appeared to influence only the sexual dimorphic behaviors of boys (Table 2). This is in accord with the viewpoint of Zambrano et al. that the sexual differentiation of the brain of males is achieved during the latter weeks of gestation, whereas the sexual differentiation of the brain of females is not achieved until 1-3 years of age [6]. Therefore, gestational information also should be considered when assessing possible postnatal influences on sexual dimorphism in boys.

In conclusion, the influence of the postnatal environment on the sexually dimorphic behavior of pre-school children should be considered as the much as the influence of prenatal stress. Generous and patient guardians, good communication, and a close relationship between the child and guardians all have a beneficial association with the development of sexual dimorphic behaviors in children. Indoor and outdoor chemical exposure also seems to contribute to disorders in sexual dimorphism. The gestational environment should be considered, particularly, in the assessment of boys. Our study highlights the importance of assessing postnatal environmental influences on sexual dimorphism during the early years of life and indicates the need for further studies with larger samples.

\section{Acknowledgements}

This study was partly supported by grants from the National Natural Science Foundation of China (No: 81372955), the Medicine and Health Science and Technology Development Project of Shandong (No: 2016WS0309) Qingdao Outstanding Health Professional Development Fund and Qingdao Medical Research and Guidance Program (No: 2014-WJZD164).

\section{References}

[1] S. H. Swan, F. Liu, M. Hines, R. L. Kruse, C. Wang, J. B. Redmon, et al., "Prenatal phthalate exposure and reduced masculine play in boys," Int. J. Androl., 2010; 33: 259-67.

[2] G. Winneke, U. Ranft, J. Wittsiepe, M. Kasper-Sonnenberg, P. Furst, U. Kramer, et al., "Behavioral sexual dimorphism in school-age children and early developmental exposure to dioxins and PCBs: a follow-up study of the Duisburg Cohort," Environ. Health Perspect., 2014; 122: 292-8.

[3] H. J. I. Vreugdenhil, F. M. E. Slijper, P. G. H. Mulder, N. Weisglas-Kuperus, "Effects of perinatal exposure to PCBs and dioxins on play behavior in dutch children at school age," Environ. Health Perspect., 2002; 110: A593-A8.

[4] M. A. Adgent, J. L. Daniels, L. J. Edwards, A. M. Siega-Riz, W. J. Rogan, "Early-Life Soy Exposure and Gender-Role Play Behavior in Children," Environ. Health Perspect., 2011; 119: 1811-6.

[5] E. S. Barrett, J. B. Redmon, C. Wang, A. Sparks, S. H. Swan, "Exposure to prenatal life events stress is associated with masculinized play behavior in girls," Neurotoxicology, 2014; 41: 20-7.

[6] E. Zambrano, C. Guzmán, G. L. Rodríguez-González, M. Durand-Carbajal, P. W. Nathanielsz, "Fetal programming of sexual development and reproductive function," Mol. Cell. Endocrinol., 2014; 382: 538-49.

[7] A. J. Ayres: Sensory integration and praxis test (SIPT), 1989. 
[8] A. Fisher, A. Bundy: Sensory integration theory. In H. Forssberg \& H. Hirschfield (Ed.). Movement disorders in children (PP 16-22). Basel: Switzerland: S. Karger Inc., 1992.

[9] R. R. Ahn, L. J. Miller, S. Milberger, D. N. McIntosh, "Prevalence of parents' perceptions of sensory processing disorders among kindergarten children," Am. J. Occup. Ther., 2004; 58: 287-93.

[10] R. A. Fabes, C. L. Martin, L. D. Hanish, "Young children's play qualities in same-, other-, and mixed-sex peer groups," Child Dev., 2003; 74:921-32.

[11] B. L. Wang, T. Takigawa, Y. Yamasaki, N. Sakano, D. H. Wang, K. Ogino, "Symptom definitions for SBS (sick building syndrome) in residential dwellings," Int. J. Hyg. Environ. Health, 2008; 211: 114-20.

[12] Y. Yamasaki, B. Wang, N. Sakano, D. Wang, T. Takigawa, "Relationship between indoor air pollutants and living environment and subjective symptoms," J. Soc. Indoor Environ., Japan, 2006; 9: 25-36.

[13] S. Wang, H. M. Ang, M. O. Tade, "Volatile organic compounds in indoor environment and photocatalytic oxidation: state of the art," Environ. Int., 2007; 33: 694-705.

[14] B. L. Wang, X. L. Li, X. B. Xu, Y. G. Sun, Q. Zhang, "Prevalence of and risk factors for subjective symptoms in urban preschool children without a cause identified by the guardian," Int. Arch. Occup. Environ. Health, 2012; 85: 483-91.

[15] G. Y. Ren, Y. F. Wang, B. M. Gu, Y. C. Shen,"Preliminary report on application of child sensory integration scale," Chin. Ment. Health J., 1994; 8: 145-7. Article in Chinese.

[16] Y. Q. Huang, Y. F. Wang, "Pretest assessment of the sensory integration scale in the preschool children aged 3-6 years," Chin. Ment. Health J., 1997; 11: 269-71. Article in Chinese.

[17] S. Golombok, J. Rust, "The Pre-School Activities Inventory: A standardized assessment of gender role in children," Psychol. Assess., 1993; 5: 131-6.

[18] Y. H. Yang, P. P. Cheng, Q. Zhang, X. Jia, S. T. Pang, B. L. Wang, "Environmental factors influencing the behavioral sexual dimorphism in preschool-aged children," Chin. J. Public Health, 2015; 31: 1116-8.

[19] Y. S. Du, L. Y. Su, "Pilot study for the assessment of the sexual type behavior in preschool-aged children," Chin. J. Clin. Psych., 1995; 3: 20-2.

[20] J. L. Luby, D. M. Barch, A. Belden, M. S. Gaffrey, R. Tillman, C. Babb, et al., "Maternal support in early childhood predicts larger hippocampal volumes at school age," Proc. Natl. Acad. Sci. U. S. A., 2012; 109: 2854-9.

[21] S. Schneider, S. Brassen, U. Bromberg, T. Banaschewski, P. Conrod, H. Flor, et al., "Maternal interpersonal affiliation is associated with adolescents' brain structure and reward processing," Translat. psych., 2012; 2: e182.
[22] P. Grandjean, P. J. Landrigan, "Developmental neurotoxicity of industrial chemicals," Lancet, 2006; 368: 2167-78.

[23] S. C. Edwards, W. Jedrychowski, M. Butscher, D. Camann, A. Kieltyka, E. Mroz, et al., "Prenatal exposure to airborne polycyclic aromatic hydrocarbons and children's intelligence at 5 years of age in a prospective cohort study in Poland," Environ. Health Perspect., 2010; 118: 1326-31.

[24] F. P. Perera, Z. Li, R. Whyatt, L. Hoepner, S. Wang, D. Camann, et al., "Prenatal airborne polycyclic aromatic hydrocarbon exposure and child IQ at age 5 years," Pediatrics, 2009; 124: e195-202.

[25] B.-L. Wang, S.-T. Pang, X.-L. Zhang, X.-L. Li, Y.-G. Sun, X.-M. Lu, et al., "Levels and neurodevelopmental effects of polycyclic aromatic hydrocarbons in settled house dust of urban dwellings on preschool-aged children in Nanjing, China," Atmos. Poll. Re., 2014; 5:292-302.

[26] K. E. Rakkestad, C. J. Dye, K. E. Yttri, J. A. Holme, J. K. Hongslo, P. E. Schwarze, et al., "Phthalate levels in Norwegian indoor air related to particle size fraction," J. Environ. Monit., 2007; 9: 1419-25.

[27] L. G. Costa, G. Giordano, S. Tagliaferri, A. Caglieri, A. Mutti, "Polybrominated diphenyl ether (PBDE) flame retardants: environmental contamination, human body burden and potential adverse health effects," Acta Biomed., 2008; 79: 172-83.

[28] B. Eskenazi, A. R. Marks, A. Bradman, K. Harley, D. B. Bart, C. Johnson, et al., "Organophosphate pesticide exposure and neurodevelopment in young Mexican-American children," Environ. Health Perspect., 2007: 792-8.

[29] F. P. Perera, V. Rauh, R. M. Whyatt, W. Y. Tsai, D. Tang, D. Diaz, et al., "Effect of prenatal exposure to airborne polycyclic aromatic hydrocarbons on neurodevelopment in the first 3 years of life among inner-city children," Environ. Health Perspect., 2006; 114: 1287-92.

[30] S. L. Schantz, J. J. Widholm, D. C. Rice, "Effects of PCB exposure on neuropsychological function in children," Environ. Health Perspect., 2003; 111:357.

[31] R. A. Rudel, L. J. Perovich, "Endocrine disrupting chemicals in indoor and outdoor air," Atmos. Environ. (1994), 2009; 43:170-81.

[32] P. P. Cheung, A. M. Siu, "A comparison of patterns of sensory processing in children with and without developmental disabilities," Res. Dev. Disabil., 2009; 30: 1468-80.

[33] W. Dunn, "Supporting children to participate successfully in everyday life by using sensory processing knowledge," Infants \& Young Children, 2007; 20: 84-101.

[34] L. J. Miller, M. E. Anzalone, S. J. Lane, S. A. Cermak, E. T. Osten, "Concept evolution in sensory integration: a proposed nosology for diagnosis," Am. J. Occup. Ther., 2007; 61: 135-40. 